University of Wollongong

Research Online

Faculty of Science, Medicine and Health -

Papers: Part B

Faculty of Science, Medicine and Health

$1-1-2018$

\title{
The influence of incubation temperature on phenotype of Australian Painted Dragons (Ctenophorus pictus)
}

Alexander Hansson

University of Gothenburg

Mats M. Olsson

University of Gothenburg, molsson@uow.edu.au

Follow this and additional works at: https://ro.uow.edu.au/smhpapers1

\section{Publication Details Citation}

Hansson, A., \& Olsson, M. M. (2018). The influence of incubation temperature on phenotype of Australian Painted Dragons (Ctenophorus pictus). Faculty of Science, Medicine and Health - Papers: Part B.

Retrieved from https://ro.uow.edu.au/smhpapers1/983 


\title{
The influence of incubation temperature on phenotype of Australian Painted Dragons (Ctenophorus pictus)
}

\author{
Abstract \\ Incubation temperature is one of the most studied factors driving phenotypic plasticity in oviparous \\ reptiles and has been shown to affect a wide variety of traits including body size, shape, and \\ performance. Thermal regimes during embryogenesis might therefore have direct consequences on \\ fitness, potentially even shaping population trajectories. These effects are likely strongest in short-lived \\ species where even temporary temperature-induced differences in body size or shape might have \\ adaptive significance. We investigated the effects of incubation temperature on the body size and shape \\ of hatchling Australian Painted Dragons (Ctenophorus pictus). Eggs incubated at low temperature \\ required a longer incubation period, but produced hatchlings of greater body mass. However, no effect of \\ temperature was found on the structural dimensions of hatchlings. These results might be explained by \\ an increased absorption of water by the developing embryo during the prolonged incubation period. A \\ greater water content might increase early-life desiccation tolerance in this short-lived lizard inhabiting \\ arid and semiarid environments. Egg mass, however, had the strongest effect on hatchling phenotype, \\ with larger eggs producing larger hatchlings. Furthermore, there was a seasonal effect on yolk allocation, \\ with eggs laid earlier being larger than those laid later. Our results indicate that yolk allocation is the most \\ important factor affecting hatchling phenotype in this species, while temperature mainly affects embryo \\ developmental rate and likely has an indirect effect on hatchling water content.
}

\section{Publication Details}

Hansson, A. \& Olsson, M. (2018). The influence of incubation temperature on phenotype of Australian Painted Dragons (Ctenophorus pictus). Herpetologica, 74 (2), 146-151. 
1 Title page

4 The Influence of Incubation Temperature on Phenotype of Australian Painted

\section{Dragons (Ctenophorus pictus)}

6

Department of Biological and Environmental Sciences, University of Gothenburg,

10 Medicinaregatan 18, SE 41390 Gothenburg, Sweden

${ }^{1}$ CORRESPONDENCE: e-mail, hansson.h.alexander@gmail.com

${ }^{2}$ Present AdDress: Box 463, SE 40530 Gothenburg, Sweden 
ABSTRACT: Incubation temperature is one of the most studied factors driving phenotypic

17 plasticity in oviparous reptiles and has been shown to affect a wide variety of traits including

18 body size, shape and performance. Thermal regimes during embryogenesis may therefore have

19 direct consequences on fitness, potentially even shaping population trajectories. These effects are

20 likely strongest in short-lived species where even temporary temperature-induced differences in

21 body size or shape may have adaptive significance. We investigated the effects of incubation

22 temperature on the body size and shape of hatchling Australian Painted Dragons (Ctenophorus

23 pictus). Eggs incubated at low temperature required a longer incubation period, but produced

24 hatchlings of greater body mass. However, no effect of temperature was found on the structural

25 dimensions of hatchlings. These results may be explained by an increased absorption of water by

26 the developing embryo during the prolonged incubation period. A greater water content may

27 increase early life desiccation tolerance in this short-lived lizard inhabiting arid and semi-arid

28 environments. Egg mass, however, had the strongest effect on hatchling phenotype, with larger

29 eggs producing larger hatchlings. Furthermore, there was a seasonal effect on yolk allocation,

30 with eggs laid earlier being larger than those laid later. Our results indicate that yolk allocation is

31 the most important factor affecting hatchling phenotype in this species, while temperature mainly

32 affects embryo developmental rate and likely has an indirect effect on hatchling water content.

34 Key words: Agamidae; Australian lizard; Egg; Hatchling phenotype; Phenotypic

35 plasticity; Seasonality; Water content; Yolk allocation

ENVIRONMENTALLY induced phenotypic variation (phenotypic plasticity) is common in 
occur at any stage during an organism's life. However, phenotypic plasticity during the egg stage

41 in oviparous reptiles may be of particular importance as embryogenesis and hatching are likely to

42 be under strong selection (Lindström 1999; Shine and Olsson 2003). The hatchling stage being

43 one of those under the strongest selection in the wild, with often more than $80-90 \%$ mortality

44 (Olsson and Madsen 2001). One of the most studied environmental factors known to drive

45 phenotypic plasticity at this stage of development is incubation temperature (Deeming 2004),

46 because embryos lack or have very limited ability to behaviorally thermoregulate (but see

47 behavioral thermoregulation in turtle embryos, Du et al. 2011; Zhao et al. 2013). This obviously

48 changes after hatching, when the neonate can exploit ambient thermal heterogeneity to control

49 their body temperature (Stevenson 1985) within relatively narrow ranges (Huey et al. 2012).

50 Eggs, however, are clearly immobile, forcing them to develop at ambient thermal regimes

51 predetermined by the mother's nest-site selection. The site of oviposition may therefore be a way

52 for the mother to indirectly manipulate the offspring's phenotype by exposing the eggs to certain

53 temperatures (Shine and Harlow 1996).

54 There is extensive experimental work on how incubation temperature may induce

55 phenotypic variation in the offspring. Incubation temperature has, in oviparous reptile hatchlings,

56 been shown to modify the expression of several phenotypic traits, such as body size, body shape,

57 sex, locomotor performance, pigmentation, and anti-predator behavior (Deeming 2004). Recent

58 studies even suggest that incubation temperature may affect brain development and thus also

59 cognitive ability (Amiel and Shine 2012; Amiel et al. 2016). All of these phenotypic traits are

60 likely closely linked to the fitness of both hatchling and adult reptiles, and thus subjected to

61 selection. Growing evidence shows that temperature-dependent effects during incubation on

62 hatchling phenotype may be species-specific (Braña and Ji 2000; Shine 2004; Telemeco et al.

63 2010; Ballen et al. 2015), making generalizations difficult. A recent meta-analysis examining the 
64 effects of incubation temperature on phenotype and survival in reptiles found that temperature

65 most strongly and widely affected incubation duration and survival (Noble et al. 2017). However,

66 the study also recognized the unpredictability of effects, especially in Squamata, which highlights

67 the importance of examining effects on specific species, rather than generalizing. The choice of

68 experimental incubation temperature should, if possible, be selected based on some form of

69 putative knowledge such as temperature in natural nests, optimal incubation temperature, or one

70 of these two combined with future predictions of climate change within a reasonable time-frame,

71 if the goal is to make evolutionary inferences of natural populations.

72 Here, we used Australian Painted Dragons (Ctenophorus pictus) to examine the variation

73 in hatchling phenotypes associated with incubation temperature, with the aim of identifying its

74 effects on incubation duration, hatching success, hatchling size and hatchling shape. No previous

75 study has examined effects of incubation temperature on any phenotypic trait except sex (Harlow

76 2004; Uller et al. 2006) in this species. However, based on similar studies in other species,

77 incubation duration is expected to decrease with temperature and hatchling success may either

78 decrease with temperature or be unaffected (Chen and Ji 2002; Lu et al. 2009; Esquerré et al.

79 2014; reviewed in Noble et al. 2017). The phenotypic traits examined in this study were chosen

80 because previous work has identified their links to components of fitness (Flatt et al. 2001;

81 Rodríguez-Díaz et al. 2010). 
88 respect to head color, and typically occur in three different head color morphs (red, orange and

89 yellow). These lizards are short-lived, with only approximately $10 \%$ live past their first year, and,

90 especially red males, are highly territorial (Olsson et al. 2007). Adult Painted Dragons used in

91 this study were caught by noose or by hand in Yathong Nature Reserve, New South Wales,

92 Australia $\left(145^{\circ} 35^{\prime} \mathrm{E} ; 32^{\circ} 35^{\prime} \mathrm{S}\right.$; datum = GDA94). Lizards were housed in mating pairs in cages

$93(50 \mathrm{~L} \times 40 \mathrm{~W} \times 35 \mathrm{H} \mathrm{cm})$ with a sand substrate and a $40 \mathrm{~W}$ spotlight at one end and rocks to

94 allow thermoregulation and shelter. The room temperature fluctuated daily between $15^{\circ} \mathrm{C}$ and

$9525^{\circ} \mathrm{C}$ to simulate natural conditions. Additionally, a small 'sand dune' was added to all cages and

96 was continuously moistened to act as a favorable oviposition site. They were fed with a variation

97 of crickets and meal worms dusted daily with calcium and weekly with multivitamins, and misted

98 daily. Males were constantly kept with the females during the entire duration of the experiment as

99 their presence is required for successful fertilization (Uller and Olsson 2005). The study took

100 place between European Autumn 2016 and Spring 2017.

101

\section{Egg Collection and Incubation}

103 Females produced $1-4$ clutches with an average of $1.63 \pm 0.20$ (mean $\pm \mathrm{SE}, n=19$ ) clutches with

104 on average $3.67 \pm 0.27$ (mean $\pm \mathrm{SE}, n=30$ ) eggs per clutch. Cages were checked daily for

105 recently laid clutches. Laid fertilized eggs ( $n=110$ from 19 females) were cleaned from sand and

106 moisture, weighed to the nearest $0.001 \mathrm{~g}$ and placed individually in $125 \mathrm{~mL}$ disposable plastic

107 cups half-buried in moist vermiculite (1:5, water:vermiculate volume) filling a third of the cup.

108 The cups were sealed with plastic cling wrap and a rubber band to prevent moisture loss and

109 placed in incubators set at constant $28^{\circ} \mathrm{C}, 30^{\circ} \mathrm{C}$ and $32^{\circ} \mathrm{C}$. There are no data on the thermal

110 profiles of the natural nests of $C$. pictus, and treatment temperatures were chosen based on the

111 previously suggested ideal incubation temperature of $30^{\circ} \mathrm{C}$ (Olsson et al. 2007). The three 
112 incubation temperatures, however, all fall within the natural thermal range of nests in situ (M.

113 Olsson, personal observation). Here, we applied constant incubation temperatures which may be

114 problematic as it has been argued that natural nests experience diel temperature fluctuations

115 (reviewed in Booth 2006). Although while studies have found that fluctuating temperatures may

116 influence hatchlings differently than constant temperatures, these effects are often limited to

117 incubation duration and locomotor performances, rather than morphological traits (Ashmore and

118 Janzen 2003; Ji et al. 2007; Du and Shine 2010; Li et al. 2014), which is the focus of this study.

119 The true temperature in the incubators was measured using HOBO H08 data loggers (Onset

120 Computer Corporation, Bourne, MA) throughout the entire duration of the experiment (191

121 days). We used a split-clutch design in which eggs from a single clutch were allocated equally

122 across the three temperature treatments. The cups were rotated among three shelves in each

123 incubator every two weeks to minimize the effect of thermal gradients inside the incubator.

124 Additionally, water was added to each cup during this rotation to account for the small, however

125 evident, loss of water through evaporation (about $1 \mathrm{~g}$ per week). Eggs were then checked daily

126 for pipping and dead eggs were removed.

129 On the day of hatching, hatchlings were blotted dry and brushed clean of vermiculite and

130 weighed to the nearest $0.001 \mathrm{~g}$. The sex of hatchlings was determined by hemipenal

131 transillumination in males (Brown 2009) after a few weeks, as hemepenes were easier to identify

132 at this stage. Hatchlings that died prior to sexing were frozen, however, some deceased offspring

133 could unfortunately not be sexed due to the compromised tissue quality and lack of

134 vascularization on which transillumination sexing depends. Importantly, mortality was not sex-

135 specific (see results) and hence does not explain our results. Size measurements were taken using 
136 a digital caliper of total length, SVL, tail length, abdomen length, head width, head length, and

137 right side: forelimb length and hind limb length (Fig. 1), all to the nearest $0.03 \mathrm{~mm}$. The duration

138 of incubation, measured as the number of days to pipping, was recorded for each egg. At the end

139 of the experiment the hatchlings were recruited to the lab population and thus determination of

140 dry mass, tissue composition and residual yolk, requiring euthanasia, were not possible.

All models were fitted in R v3.3.2 (R Core Team 2016) following a linear mixed model

144 (LMM) and generalized linear mixed model (GLMM) approach using the functions lmer and

145 glmer from the lme4 package (Bates et al. 2015) with female identity as random effect. Estimates

146 of $P$-values were obtained using parametric likelihood ratio bootstrapping with the $P$ Bmodcomp

147 function from the pbkrtest package (Ulrich and Søren 2014). All bootstrap analyzes were based

148 on 10,000 iterations. The effects of incubation temperature and egg mass on hatching success,

149 incubation duration and phenotypic traits were examined. We also investigated the possibility for

150 sexual dimorphism in the hatchlings and sex effects on incubation duration. The main effects

151 were corrected for the effects of egg mass and temperature, with a $P$-to-enter set at 0.15 (Bursac

152 et al. 2008). All data met the requirements of the statistical tests except a deviation from a normal

153 distribution in the abdomen lengths and incubation duration, which were corrected by log

154 transformations. The incubation temperature of each egg was calculated as an average of the

155 logger data from each incubation period. No interactions were found among incubation

156 temperature, initial egg mass and sex, and were therefore excluded from the analyzes. 
160 The actual temperatures in the incubators, measured by temperature loggers, were in the $28^{\circ} \mathrm{C}$,

$16130^{\circ} \mathrm{C}$ and $32^{\circ} \mathrm{C}$ treatment $\left(\right.$ mean $\left.{ }^{\circ} \mathrm{C} \pm \mathrm{SE}\right): 27.9 \pm 0.002(n=13,337), 29.9 \pm 0.004(n=10,055)$

162 and $31.8 \pm 0.004(n=15,453)$, respectively. Incubation duration was strongly affected by

163 incubation temperature (Table 2), with hatchlings emerging earlier at higher temperatures. The

164 average incubation periods differed over two weeks between $28^{\circ} \mathrm{C}$ and $32^{\circ} \mathrm{C}$ (Table 1). The

165 duration of incubation was also strongly affected by initial egg mass, with larger eggs hatching

166 later. No significant effect of sex on incubation duration was found (Table 2). Overall hatching

167 success was $60 \%$ (66 of 110) and did not differ among incubation temperatures $\left(\chi^{2}=1.21, P=\right.$

$1680.28, n=110)$ or initial egg mass $\left(\chi^{2}=0.13, P=0.74, n=110\right)$. Date of oviposition, however,

169 affected hatchling success $\left(\chi^{2}=6.42, P=0.013, n=110\right)$, with higher egg mortality later in the

170 season. There was a rather high egg mortality, however, it is difficult to differentiate fertile from

171 infertile eggs in Painted Dragons and we have described before that fertility is labile in this

172 species and requires continuous male presence (Uller and Olsson 2005). The degree to which

173 females have responded to male presence is hard to evaluate and was likely the reason for the low

174 fertilization rate. Thus, fertility is hard to establish and the majority of unhatched eggs were in

175 fact probably never fertile in the first place.

176

expectedly, produced hatchlings with larger body size (Table 1), including a significant effect on

182 body mass, total length, tail length, head length, head width and hind limb length (Table 2). SVL

183 also followed the same trend, however, with a significance value just above the 0.05 threshold. 
184 Furthermore, eggs laid earlier in the breeding season were larger than those laid later $\left(\chi^{2}=23.12\right.$,

$185 P<0.001, n=110)$. The date of oviposition, however, had no effect on any phenotypic trait

186 measured and was therefore excluded from the morphological analyses. Sex did not significantly

187 affect any of the phenotypic traits measured, except forelimb length (Table 2). Post-hatching 188 mortality was not sex-specific $\left(\chi^{2}=0.32, P=0.60, n=47\right)$.

\section{DISCUSSION}

191 Consistent with studies of other oviparous reptiles (reviewed in Deeming 2004; Deeming

192 and Ferguson 1991), we show that higher incubation temperatures result in shorter incubation

193 durations in Australian Painted Dragons. Variation in incubation duration are most often driven

194 by variation in rate of embryogenesis, easily explained by thermal effects $\left(Q_{10}\right)$ on chemical

195 activities, including metabolic rate. Incubation temperature has also been shown to affect

196 hatchling size in many reptiles, with generally 'larger' in terms of skeletal dimensions, but not

197 always heavier, hatchlings at lower incubation temperature (reviewed in Deeming 2004; Booth

198 2006). Our results, however, showed a reversed effect, with lower incubation temperature

199 producing heavier, rather than structurally larger, hatchlings. It is possible that this deviation

200 reflects a species-specific response associate to the particular ecological contingencies of the

201 desert-like habitat occupied by $C$. pictus. The majority of studies on developmental biology in

202 reptiles are focused on reptiles living in habitats with more abundant resources (e.g. reviewed in

203 Booth 2006). In the case of C. pictus, selection may favor less developed hatchings, in terms of

204 structural size, with a larger residual yolk reserve working as a buffer against desiccation during

205 early life in dry environments (Packard 1991). Although we did not examine residual yolk mass,

206 residual yolk was released from the cloacae during measurements of hatchlings from the high 
207 temperature treatment. This was not observed in the low temperature hatchlings, suggesting a

208 higher abundance of residual yolk in high temperature hatchlings.

209 The limited effect of incubation temperature on the morphology and hatching success in

210 this species indicate a robustness to variations in ambient temperature and thus possibly against

211 climate change. However, morphology is only one factor that may be influenced by incubation

212 temperature. It would be necessary to examine the effects of incubation temperature on sex ratio,

213 locomotor performance, and post-hatch growth, survival and reproductive success to determine

214 how this lizard may cope with climate change.

215 The body mass of all hatchlings exceeded the initial egg mass at oviposition, indicating

216 that eggs absorbed water during the embryonic development. This has been suggested to be the

217 case in many small lizard eggs (Tracy 1980; Vleck 1991) and clear evidence for this has been

218 recorded (Ji and Braña 1999; Booth et al. 2000; Ji and Zhang 2001). The reproductive strategy of

219 producing eggs with lower water content will enhance maternal fitness: (1) in habitats where

220 water is a limiting resource for adults, but available for absorption by eggs; (2) in species where

221 bulky eggs would result in a decrement in maternal fitness; and/or (3) allowing for the production

222 of large clutches without the gravid female requiring a great volume (Tracy 1980). These features

223 are congruent with the life-history of Australian Painted Dragons, which inhabits relatively arid

224 niches, where moisture can be absorbed by eggs from the soil. It produces clutches of a size that

225 clearly impedes motility, where larger eggs with a higher water content would likely result in a

226 decrement in maternal fitness. The increased mass of hatchlings in this study, compared to the

227 initial egg mass indicate that water absorbed by the egg is not only used for embryo development,

228 but is incorporated within the hatchling, effectively increasing its body mass (Ji and Braña 1999).

229 Longer incubation periods allow for more water to be absorbed by the egg (Booth et al. 2000),

230 which has been shown to increase the water content and thus also the body mass of hatchlings 
231 (Packard et al. 1988; Finkler 1999). The increased body mass in hatchlings incubated at low

232 temperature may therefore simply be due to a greater water content (Ji and Braña 1999) as an

233 effect of longer incubation periods and thus an indirect effect of incubation temperature.

234 Unfortunately, water content can only be analyzed by assessing dry mass which was not done in

235 this study because the fate of the hatchlings was not euthanasia, but recruitment. Nevertheless,

236 hatchlings with greater water content will, when water is scarce, be able to survive longer and

237 loose more water before being adversely affected by desiccation (Finkler 1999). This additional

238 water content may be of major importance for this lizard inhabiting semi-arid environments,

239 typically with hot summers and unpredictable rainfall.

240 Regardless of possible temperature effects on hatchling phenotype, the strongest

241 contributor to hatchling morphology remains maternal yolk allocation (Ferguson and Snell 1986;

242 Deeming and Ferguson 1989; Nelson et al. 2004), affecting size and shape of hatchlings. The

243 yolk has a fundamental influence on the development from zygote to complete hatchling, not

244 only determining how much resources are available for growth and maintenance during

245 embryogenesis, but any surplus may affect hatchling survival when taking the form of residual

246 yolk and fat reserves (reviewed in Congdon 1989). There was also a significant effect of

247 oviposition date on egg mass and hatchling success, with larger eggs and higher embryo survival

248 earlier in the season. Eggs laid earlier in the season are likely of better quality than those laid later

249 due to a greater maternal yolk allocation. This may simply be a result of a seasonally declining

250 maternal condition (Olsson and Shine 1997). Similarly, Warner and Shine 2007 found that the

251 fitness of juvenile jacky dragon lizards depends on seasonal timing of hatching, with early

252 hatched individuals experiencing greater survival rates and chance of reproducing in their first

253 year. They argue that early clutches provide greater fitness returns which may create a selection

254 pressure on the mother to reproduce as early as possible in the season and to allocate more 
255 resources toward these early clutches. It is possible that a similar selection pressure could be

256 acting on $C$. pictus.

258 Acknowledgments. - We are grateful for funding support from the Australian Research Council 259 (MO) and the Swedish Science Council (MO). Special thanks to T. Slight for maintenance and

260 feeding of the lizards. AH collected and analyzed the data, and drafted the manuscript. AH and

261 MO designed the experiment and contributed to revisions of the manuscript. This research was

262 conducted under a scientific research permit (identification number: 001066) issued by the

263 Animal Ethics Committee at the University of Gothenburg, Sweden. Two anonymous reviewers

264 provided constructive criticism on an earlier version of the manuscript. We declare no competing 265 interests.

\section{LITERATURE CITED}

268 Amiel, J.J., S. Bao, and R. Shine. 2016. The effects of incubation temperature on the

269 development of the cortical forebrain in a lizard. Animal Cognition 20:117-125.

270 Amiel, J.J., and R. Shine. 2012. Hotter nests produce smarter young lizards. Biology Letters $271 \quad 8: 372-374$.

272 Ashmore, G.M., and F.J. Janzen. 2003. Phenotypic variation in smooth softshell turtles (Apalone 273 mutica) from eggs incubated in constant versus fluctuating temperatures. Oecologia $274 \quad 134: 182-188$.

275 Ballen, C.J., R. Shine, and M. Olsson. 2015. Developmental plasticity in an unusual animal: 276 the effects of incubation temperature on behavior in chameleons. Behaviour 152:1307-1324.

277 Bates, D.M., M. Machler, B.M. Bolker, and S.C. Walker. 2015. Fitting linear mixed-effects 278 models using lme4. Journal of Statistical Software 67:1-48. 
279 Booth, D.T. 2006. Influence of incubation temperature on hatchling phenotype in

280 reptiles. Physiological and Biochemical Zoology 79:274-281.

281 Booth, D.T., M.B. Thompson, and S. Herring. 2000. How incubation temperature influences the

282 physiology and growth of embryonic lizards. Journal of Comparative Physiology B 170:269283276.

284 Braña, F., and X. Ji. 2000. Influence of incubation temperature on morphology, locomotor 285 performance, and early growth of hatchling wall lizards (Podarcis muralis). Journal of 286 Experimental Zoology 286:422-433.

287 Brown, D. 2009. Hemipenal transillumination as a sexing technique in varanids. Baiwak 3:26-29.

288 Bursac, Z., C.H. Gauss, D.K. Williams, and D. Hosmer. 2008. Purposeful selection of variables macro for logistic regression. Source Code for Biology and Medicine 3:17.

290 Chen, H., and X. Ji. 2002. The effects of thermal environments on duration of incubation, 291 hatching success and hatchling traits in a colubrid snake Rhabdophis tigrinus lateralis 292 (Boie). Acta Ecologica Sinica 22:1850-1858.

293 Congdon, J.D. 1989. Proximate and evolutionary constraints on energy relations of 294 reptiles. Physiological Zoology 62:356-373.

295 Deeming, D.C. 2004. Post-hatching phenotypic effects of incubation in reptiles. Pp. 229-251 in 296 Reptilian Incubation: Environment, Evolution and Behaviour (D.C. Deeming, ed.).

297 Nottingham University Press, UK.

298 Deeming, D.C., and M.W. Ferguson. 1989. Effects of incubation temperature on growth and 299 development of embryos of Alligator mississippiensis. Journal of Comparative Physiology B $300 \quad 159: 183-193$.

301 Deeming, D.C., and M.W. Ferguson. 1991. Physiological effects of temperature on embryonic 302 development in reptiles and birds. Pp. 147-171 in Egg Incubation: Its Effects on Embryonic 
303 Development in Birds and Reptiles (D.C. Deeming and M. Ferguson, eds.). Cambridge

$304 \quad$ University Press, UK.

305 Du, W., and R. Shine. 2010. Why do the eggs of lizards (Bassiana duperreyi: Scincidae) hatch

306 sooner if incubated at fluctuating rather than constant temperatures?. Biological Journal of 307 the Linnean Society 101:642-650.

308 Du, W., B. Zhao, Y. Chen, and R. Shine. 2011. Behavioral thermoregulation by turtle embryos.

309 Proceedings of the National Academy of Sciences 108:9513-9515.

310 Esquerré, D., J.S. Keogh, and L.E. Schwanz. 2014. Direct effects of incubation temperature on

311 morphology, thermoregulatory behaviour and locomotor performance in jacky dragons

312 (Amphibolurus muricatus). Journal of Thermal Biology 43:33-39.

313 Ferguson, G.W., and H.L. Snell. 1986. Endogenous control of seasonal change of egg, hatchling, 314 and clutch size of the lizard Sceloporus undulatus garmani. Herpetologica 42:185-191.

315 Finkler, M.S. 1999. Influence of water availability during incubation on hatchling size, body

316 composition, desiccation tolerance, and terrestrial locomotor performance in the snapping

317 turtle Chelydra serpentina. Physiological and Biochemical Zoology 72:714-722.

318 Flatt, T., R. Shine, P.A. Borges-Landaez, S.J. Downes. 2001. Phenotypic variation in an

319 oviparous montane lizard (Bassiana duperreyi): the effects of thermal and hydric incubation 320 environments. Biological Journal of Linnean Society 74:339-350.

321 Harlow, P.S. 2004. Temperature-dependent sex determination in lizards. Pp. 42-52 in

322 Temperature-Dependent Sex Determination in Vertebrates (N. Valenzuela and V. Lance, 323 eds.). Smithsonian Institution Press, USA.

324 Huey, R.B., M.R. Kearney, A.K. Krockenberger, J.A.M. Holtum, M. Jess, and S.E. Williams.

325 2012. Predicting organismal vulnerability to climate warming: roles of behaviour, 
326 physiology and adaptation. Philosophical Transactions of the Royal Society B 367:1665-

$327 \quad 1679$.

328 Ji, X., and F. Braña. 1999. The influence of thermal and hydric environments on embryonic use

329 of energy and nutrients, and hatchling traits, in the wall lizards (Podarcis

330 muralis). Comparative Biochemistry and Physiology A 124:205-213.

331 Ji, X., J. Gao, and J. Han. 2007. Phenotypic responses of hatchlings to constant versus fluctuating

332 incubation temperatures in the multi-banded krait, Bungarus multicintus

333 (Elapidae). Zoological Science 24:384-390.

334 Ji, X., and C. Zhang. 2001. Effects of thermal and hydric environments on incubating eggs,

335 hatchling success, and hatchling traits in the Chinese skink (Eumeces chinensis). Acta

336 Zoologica Sinica 47:256-265.

337 Lindström, J. 1999. Early development and fitness in birds and mammals. Trends in Ecology \& $338 \quad$ Evolution 14:343-348.

339 Li, H., Z. Zhou, G. Ding, and X. Ji. 2011. Fluctuations in incubation temperature affect

340 incubation duration but not morphology, locomotion and growth of hatchlings in the sand

$341 \quad$ lizard Lacerta agilis (Lacertidae). Acta Zoologica 94:11-18.

342 Lu, H., R. Hu, and X. Ji. 2009. The variance of incubation temperatures does not affect the

343 phenotype of hatchlings in a colubrid snake, Xenochrophis piscator. Journal of Thermal

$344 \quad$ Biology 34:138-143.

345 Nelson, N.J., M.B. Thompson, S. Pledger, S.N. Keall, and C.H. Daugherty. 2004. Egg mass

346 determines hatchling size, and incubation temperature influences post-hatching growth, of

347 tuatara Sphenodon punctatus. Journal of Zoology 263:77-87. 
348 Noble, D.W., V. Stenhouse, and L.E. Schwanz. 2017. Developmental temperatures and

349 phenotypic plasticity in reptiles: a systematic review and meta-analysis. Biological Reviews

350 DOI: http://dx.doi.org/10.1111/brv.12333

351 Olsson, M., M. Healey, E. Wapstra, T.S. Schwartz, N. Lebas, and T. Uller. 2007. Mating system

352 variation and morph fluctuations in a polymorphic lizard. Molecular Ecology 16:5307-5315.

353 Olsson, M., and T. Madsen. 2001. Between-year variation in determinants of offspring survival in

354 the sand Lizard, Lacerta agilis. Functional Ecology 15:443-450.

355 Olsson, M. and R. Shine. 1997. The seasonal timing of oviposition in sand lizards (Lacerta

356 agilis): why early clutches are better. Journal of Evolutionary Biology 10:369-381.

357 Packard, G.C. 1991. The physiological and ecological importance of water to embryos of

358 oviparous reptiles. Pp. 219 in Egg Incubation: Its Effects on Embryonic Development in

359 Birds and Reptiles (D.C. Deeming, and M.W. Ferguson, eds.). Cambridge University Press,

$360 \quad$ UK.

361 Packard, G.C., M.J. Packard, K. Miller, and T.J. Boardman. 1988. Effects of temperature and

362 moisture during incubation on carcass composition of hatchling snapping turtles (Chelydra

363 serpentina). Journal of Comparative Physiology B 158:117-125.

364 R Core Team. 2016. R: A Language and Environment for Statistical Computing. R Foundation,

$365 \quad$ Austria.

366 Rodríguez-Díaz, T., F. González, X. Ji, and F. Braña. 2010. Effects of incubation temperature on

367 hatchling phenotypes in an oviparous lizard with prolonged egg retention: are the two main

368 hypotheses on the evolution of viviparity compatible?. Zoology 113:33-38.

369 Scheiner, S.M. 1993. Genetics and evolution of phenotypic plasticity. Annual Review of

370 Ecology, Evolution and Systematics 24:35-68. 
371 Shine, R. 2004. Seasonal shifts in nest temperature can modify the phenotypes of hatchling

372 lizards, regardless of overall mean incubation temperature. Functional Ecology 18:43-49.

373 Shine, R., and P.S. Harlow. 1996. Maternal manipulation of offspring phenotypes via nest-site

$374 \quad$ selection in an oviparous lizard. Ecology 77:1808-1817.

375 Shine, R., and M. Olsson. 2003. When to be born? Prolonged pregnancy or incubation enhances

376 locomotor performance in neonatal lizards (Scincidae). Journal of Evolutionary Biology

$377 \quad 16: 823-832$.

378 Stevenson, R.D. 1985. The relative importance of behavioral and physiological adjustments

379 controlling body temperature in terrestrial ectotherms. American Nationalist 126:362-386.

380 Telemeco, R.S., R.S. Radder, T.A. Baird, and R. Shine. 2010. Thermal effects on reptile

381 reproduction: adaptation and phenotypic plasticity in a montane lizard. Biological Journal of

$382 \quad$ Linnean Society 100:642-655.

383 Tracy, C.R. 1980. Water relations of parchment-shelled lizard (Sceloporus undulatus)

$384 \quad$ eggs. Copeia 1980:478-482.

385 Uller, T., and M. Olsson. 2005. Continuous male presence required for fertilization in captive

386 painted dragons, Ctenophorus pictus. Journal of Experimental Zoology A 303:1115-1119.

387 Uller, T., B. Mott, G. Odierna, and M. Olsson. 2006. Consistent sex ratio bias of individual

388 female dragon lizards. Biology Letters 2:569-572.

389 Ulrich, H., and H. Søren. 2014. A Kenward-Roger approximation and parametric bootstrap

390 methods for tests in linear mixed models - the R package pbkrtest. Journal of Statistical

$391 \quad$ Software 59:1-30.

392 Vleck, D. 1991. Water economy and solute regulation of reptilian and avian embryos. Pp. 245-

393 in Egg Incubation: Its Effects on Embryonic Development in Birds and Reptiles (D.C.

394 Deeming and M. Ferguson, eds.). Cambridge University Press, UK. 
395 Warner, D.A. and R. Shine. 2007. Fitness of juvenile lizards depends on seasonal timing of

396 hatching, not offspring body size. Oecologia 154:65-73.

397 Zhao, B., T. Li, R. Shine, and W. Du. 2013. Turtle embryos move to optimal thermal

398 environments within the egg. Biology Letters 9:20130337. 
400 TABLE 1.-Descriptive statistics for incubation duration, mass, size and hatching success of

401 hatchlings incubated at different temperatures and differences between the sexes. ${ }^{\mathrm{a}}$

\begin{tabular}{|c|c|c|c|c|c|}
\hline \multirow[b]{2}{*}{ Trait } & \multicolumn{3}{|c|}{ Temperature } & \multicolumn{2}{|c|}{ Sex } \\
\hline & $\begin{array}{c}28^{\circ} \mathrm{C} \\
(n=25)\end{array}$ & $\begin{array}{c}30^{\circ} \mathrm{C} \\
(n=20)\end{array}$ & $\begin{array}{c}32^{\circ} \mathrm{C} \\
(n=20)\end{array}$ & $\begin{array}{c}\text { Male } \\
(n=14)\end{array}$ & $\begin{array}{l}\text { Female } \\
(n=33)\end{array}$ \\
\hline Incubation duration (days) & $56.6 \pm 0.19$ & $47.0 \pm 0.27$ & $40.9 \pm 0.25$ & - & - \\
\hline Egg mass (g) & $0.806 \pm 0.018$ & $0.781 \pm 0.018$ & $0.798 \pm 0.016$ & $0.790 \pm 0.024$ & $0.801 \pm 0.013$ \\
\hline \multicolumn{6}{|l|}{ Hachling trait } \\
\hline Mass (g) & $0.969 \pm 0.020$ & $0.935 \pm 0.021$ & $0.941 \pm 0.022$ & $0.961 \pm 0.035$ & $0.960 \pm 0.016$ \\
\hline Total length $(\mathrm{mm})$ & $69.75 \pm 0.97$ & $69.60 \pm 0.77$ & $69.85 \pm 1.02$ & $70.27 \pm 1.18$ & $69.51 \pm 0.78$ \\
\hline SVL (mm) & $28.01 \pm 0.22$ & $27.66 \pm 0.21$ & $27.73 \pm 0.28$ & $27.66 \pm 0.30$ & $27.92 \pm 0.19$ \\
\hline Tail length (mm) & $41.74 \pm 0.83$ & $41.94 \pm 0.65$ & $42.13 \pm 0.80$ & $42.61 \pm 1.01$ & $41.59 \pm 0.62$ \\
\hline Head length (mm) & $6.98 \pm 0.08$ & $7.01 \pm 0.09$ & $6.97 \pm 0.06$ & $6.82 \pm 0.09$ & $7.00 \pm 0.06$ \\
\hline Head width (mm) & $7.70 \pm 0.05$ & $7.67 \pm 0.05$ & $7.64 \pm 0.04$ & $7.67 \pm 0.06$ & $7.66 \pm 0.04$ \\
\hline Abdomen length (mm) & $10.37 \pm 0.16$ & $10.60 \pm 0.22$ & $10.53 \pm 0.16$ & $10.33 \pm 0.21$ & $10.49 \pm 0.13$ \\
\hline Forelimb length (mm) & $7.53 \pm 0.09$ & $7.33 \pm 0.06$ & $7.40 \pm 0.07$ & $7.30 \pm 0.09$ & $7.49 \pm 0.07$ \\
\hline Hind limb length (mm) & $12.68 \pm 0.12$ & $12.50 \pm 0.13$ & $12.59 \pm 0.14$ & $12.57 \pm 0.15$ & $12.66 \pm 0.09$ \\
\hline Hatching success (\%) & $67.6(25 / 37)$ & $55.6(20 / 36)$ & $56.8(21 / 37)$ & - & - \\
\hline
\end{tabular}

$402 \quad{ }^{a}$ Values are expressed as mean \pm SE for all parameters but hatchling success, shown by the

403 percentage of hatched individual (number hatched/total eggs). 
405 TABLE 2.-Results from parametric bootstrapping of linear mixed models, fitted with the lmer

406 function from the lme4 package, to examine the effects of incubation temperature, initial egg 407 mass and hatchling sex on incubation duration and morphology of hatchlings. ${ }^{\mathrm{a}}$

\begin{tabular}{|c|c|c|c|c|c|c|}
\hline \multirow[b]{2}{*}{ Trait } & \multicolumn{2}{|c|}{$\begin{array}{l}\text { Main effect of temperature } \\
\qquad(n=65)\end{array}$} & \multicolumn{2}{|c|}{$\begin{array}{l}\text { Main effect of egg mass } \\
\qquad(n=65)\end{array}$} & \multicolumn{2}{|c|}{$\begin{array}{l}\text { Main effect of sex } \\
\qquad(n=47)\end{array}$} \\
\hline & $\chi^{2}(\mathrm{df}=1)$ & $P$ & $\chi^{2}(\mathrm{df}=1)$ & $P$ & $\chi^{2}(\mathrm{df}=1)$ & $P$ \\
\hline Incubation duration & 249.01 & $<0.001 *$ & 6.61 & $0.017 *$ & 0.70 & 0.427 \\
\hline \multicolumn{7}{|l|}{ Morphology } \\
\hline Mass & 4.70 & $0.032 *$ & 44.67 & $<0.001 *$ & 0.58 & 0.468 \\
\hline Total length & 0.19 & 0.670 & 5.22 & $0.027^{*}$ & 0.02 & 0.890 \\
\hline SVL & 0.47 & 0.501 & 4.02 & 0.056 & 3.08 & 0.089 \\
\hline Tail length & 0.48 & 0.501 & 4.32 & $0.045^{*}$ & 0.08 & 0.784 \\
\hline Abdomen length & 1.13 & 0.301 & 2.93 & 0.113 & 0.41 & 0.537 \\
\hline Head length & 0.46 & 0.513 & 7.78 & $0.008^{*}$ & 1.58 & 0.229 \\
\hline Head width & 0.35 & 0.560 & 27.11 & $<0.001 *$ & 0.06 & 0.819 \\
\hline Forelimb length & 1.18 & 0.283 & 0.00 & 1.000 & 5.01 & $0.028^{*}$ \\
\hline Hind limb length & 0.34 & 0.567 & 6.29 & $0.018^{*}$ & 0.31 & 0.593 \\
\hline
\end{tabular}

$408{ }^{\mathrm{a}}$ Initial egg mass and temperature are included as covariates when significant at the 0.15 level.

409 Statistics based on 10,000 bootstrap iterations.

$410 *$ Significant value $(P<0.05)$. 


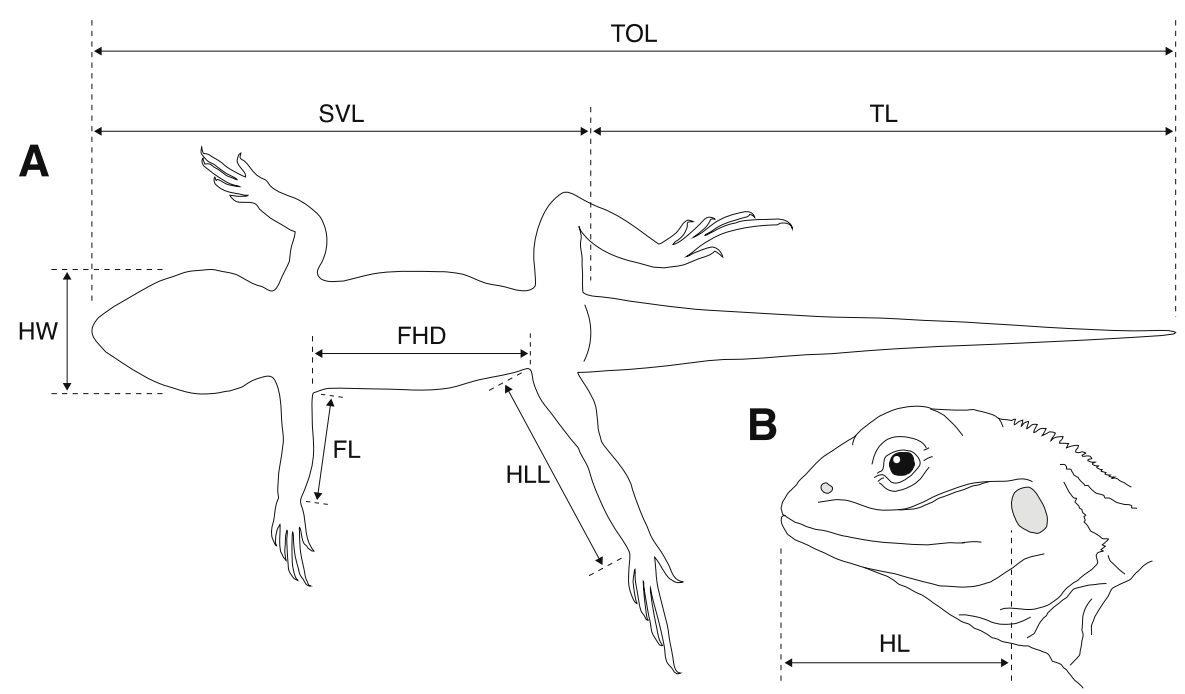

412 Fig. 1.-Morphometric measurements used for analysis. (A) Ventral and (B) lateral view 413 of the body plan and head of Ctenophorus pictus, showing measurements recorded. Total length

414 (TOL), tip of snout to tip of tail; snout-vent length (SVL), tip of snout to anterior end of vent; tail 415 length (TL), anterior end of vent to tip of tail; fore-hind limb distance (FHD), distance between 416 the inner insertion of the forelimb to the inner insertion of the hind limb, here used as abdomen 417 length; head length (HL), ventral measurement from tip of snout to the anterior end of the 418 external ear; head width (HW), maximum width of head anterior to external ear; forelimb length 419 (FL), from insertion of forelimb to the proximal end of manus; hind limb length (HLL), from 420 insertion of hind limb to proximal end of foot. 